\title{
Multilinear paraproducts revisited
}

\author{
Loukas Grafakos, Danqing He, Nigel Kalton and Mieczysław Mastyło
}

In memory of Nigel Kalton

\begin{abstract}
We prove that multilinear paraproducts are bounded from products of Lebesgue spaces $L^{p_{1}} \times \cdots \times L^{p_{m+1}}$ to $L^{p, \infty}$, when $1 \leq p_{1}, \ldots, p_{m}$, $p_{m+1}<\infty, 1 / p_{1}+\cdots+1 / p_{m+1}=1 / p$. We focus on the endpoint case when some indices $p_{j}$ are equal to 1 , in particular we obtain a new proof of the estimate $L^{1} \times \cdots \times L^{1} \rightarrow L^{1 /(m+1), \infty}$.
\end{abstract}

\section{Introduction}

Paraproducts have become tools of great use in analysis and PDEs. They are traditionally built by Littlewood-Paley square functions and may appear in different forms. Paraproducts first emerged in Bony's theory of paradifferential operators [5], which has taken a step further the pseudodifferential operator theory of Coifman and Meyer [6]. They provide important examples of operators with specific properties and have been used in significant applications, such as the proof of the $T 1$ theorem by David and Journé [7]. The relationship of paraproducts with Carleson measures and BMO is so intimate that the former have been on the forefront of research in harmonic analysis through almost a quarter century. The boundedness of paraproducts on $L^{p}$ spaces for $p>1$ is easily achieved via duality, but the extension to indices $p \leq 1$ is more delicate and was proved independently by Grafakos and Kalton [9] and by Auscher, Hofmann, Muscalu, Thiele, and Tao [1]; a different proof was given by Bényi, Maldonado, Nahmod, and Torres [2]. Hundreds of references exist on paraproducts today; of these the articles [4], [9], [13] and [14] focus on delicate boundedness properties of them. The expository article of Bényi, Maldonado, Naibo [3] presents a well-motivated introduction to paraproducts.

Multilinear paraproducts may have first appeared explicitly in the work of Yabuta [16] and later resurfaced in the work of Sato and Yabuta [15] who obtained their $L^{p}$ boundedness for $p \geq 1$. Although paraproducts fit into the class 
of multilinear Calderón-Zygmund theory, one may wonder if there are insightful direct proofs of their $L^{p}$ (resp. weak $L^{p}$ ) boundedness, especially in the difficult case $p<1$. Such proofs would take into account the specific form of paraproducts and would reflect the interplay of their intrinsic orthogonality with the orthogonality of $L^{p}$ (resp. weak $L^{p}$ ). In this work we undertake this task and we include the endpoint cases when at least one index is 1 . Our work is based on a weak type square function inequality (Lemma 1.2) recently obtained in [11], which is valid for all $0<p<\infty$. Another type of $m$-linear paraproducts built by sums of wave packets associated with dyadic intervals on the line has been studied by Lacey and Metcalfe [12] who obtained similar endpoint estimates to the ones in this article for the paraproducts built by the Littlewood-Paley operators.

We will be working on $\mathbb{R}^{d}$ for some natural number $d$. For a Schwartz function $\Phi$ we denote by $\Delta_{j}^{\Phi}$ the Littlewood-Paley operator given by convolution with the function $\Phi_{2^{-j}}(x)=2^{j d} \Phi\left(2^{j} x\right)$. We denote by $S_{j}^{\Phi}=\sum_{k \leq j} \Delta_{k}^{\Phi}$ the partial sum operator of the $\Delta_{k}^{\Phi}$ 's. For fixed smooth bumps $\Phi$ and $\Theta$ whose Fourier transforms have compact supports that do not contain the origin, we define the paraproduct operator

$$
P_{2}(f, g)=\sum_{j \in \mathbb{Z}} \sum_{k \leq j} \Delta_{j}^{\Theta}(f) \Delta_{k}^{\Phi}(g)=\sum_{j \in \mathbb{Z}} \Delta_{j}^{\Theta}(f) S_{j}^{\Phi}(g),
$$

for Schwartz functions $f, g$. This operator and its $(m+1)$-linear version is the main object of study of this paper. This is defined by

$$
P_{m+1}\left(f_{0}, f_{1}, \ldots, f_{m}\right)=\sum_{j \in \mathbb{Z}} \Delta_{j}^{\Theta}\left(f_{0}\right) S_{j}^{\Theta_{1}}\left(f_{1}\right) \cdots S_{j}^{\Theta_{m}}\left(f_{m}\right)
$$

for Schwartz functions $f_{0}, f_{1}, \ldots, f_{m}$ and smooth bumps $\Theta, \Theta_{1}, \ldots, \Theta_{m}$.

For $0<p<\infty$, we denote by $L^{p}$ the space of all measurable functions on $\mathbb{R}^{d}$ whose $p$ th power is integrable over $\mathbb{R}^{d}$ and by $L^{p, \infty}$ the space of all measurable functions $h$ that satisfy

$$
\|h\|_{L^{p, \infty}}=\sup _{\lambda>0} \lambda\left|\left\{x \in \mathbb{R}^{d}:|h(x)|>\lambda\right\}\right|^{1 / p}<\infty .
$$

Given a bump $\Psi$, we define the square function associated with $\Psi$ by

$$
\mathbf{S}^{\Psi}(f)=\left(\sum_{\ell \in \mathbb{Z}}\left|\Delta_{\ell}^{\Psi}(f)\right|^{2}\right)^{1 / 2} .
$$

We will also work with the "lacunary" square function

$$
\mathbf{S}_{q}^{\Psi}(f)=\left(\sum_{\ell \in \mathbb{Z}}\left|\Delta_{q \ell}^{\Psi}(f)\right|^{2}\right)^{1 / 2}
$$

defined for a positive integer $q$. (Notice that $\mathbf{S}_{1}^{\Psi}=\mathbf{S}^{\Psi}$.) Under very mild assumptions on $\Psi$ (such as $|\Psi(x)|+|\nabla \Psi(x)| \leq A(1+|x|)^{-d-\varepsilon}$ and $\int_{\mathbb{R}^{d}} \Psi(x) d x=0$ ), it is known that $\mathbf{S}^{\Psi}$ (also $\mathbf{S}_{q}^{\Psi}$ ) maps $L^{r}\left(\mathbb{R}^{d}\right)$ to $L^{r, \infty}\left(\mathbb{R}^{d}\right)$ for all $1 \leq r<\infty$ (see [8]). 
Finally, we denote by $\mathbf{M}$ the Hardy-Littlewood maximal operator. We recall that

$$
\sup _{j \in \mathbb{Z}}\left|\Delta_{j}^{\Theta}(f)\right|+\sup _{j \in \mathbb{Z}}\left|S_{j}^{\Theta}(f)\right| \leq C_{\Theta} \mathbf{M}(f),
$$

for all Schwartz functions $f$, for some constant $C_{\Theta}$.

The main goal of this paper is to indicate how to obtain boundedness for $P_{m+1}$ from the product of Lebesgue spaces $L^{p_{0}} \times L^{p_{1}} \times \cdots \times L^{p_{m}}$ to $L^{p, \infty}$ whenever $1 \leq p_{0}, p_{1}, \ldots, p_{m}<\infty$ and $p=\left(p_{0}^{-1}+p_{1}^{-1}+\cdots+p_{m}^{-1}\right)^{-1}$. The case $p \geq 1$ is quite easy to deal with via duality and Hölder's inequality, but the case $p<1$ is more delicate and we will focus on it. In particular, we show paraproducts map $L^{1} \times \cdots \times L^{1} \rightarrow L^{1 /(m+1), \infty}$ which is the strongest endpoint estimate concerning them.

When $m=1$ this result is known, see for instance [9], [1], [12], but the contribution of this paper is to provide a simple proof of it that does not rely on deep technical machinery (tiles, Carleson measures) and which also works for all $m \geq 1$. The following is our main result.

Theorem 1.1. Fix an integer $m \geq 1$ and smooth bumps $\Theta, \Theta_{1}, \ldots, \Theta_{m}$ whose Fourier transforms are compactly supported in $\mathbb{R}^{d} \backslash\{0\}$. For each $0 \leq k \leq m-1$ and functions $f_{j}$ in the Schwartz class of $\mathbb{R}^{d}$ define the $(m+1)$-linear paraproduct

$$
P_{m+1}^{(k)}\left(f_{0}, f_{1}, \ldots, f_{m}\right)=\sum_{j \in \mathbb{Z}}\left[\Delta_{j}^{\Theta}\left(f_{0}\right) \prod_{s=1}^{k} \Delta_{j}^{\Theta_{s}}\left(f_{s}\right) \prod_{s=k+1}^{m} S_{j}^{\Theta_{s}}\left(f_{s}\right)\right],
$$

with the understanding that when $k=0$, the first product is missing. Let $p$ be defined by $p^{-1}=p_{0}^{-1}+p_{1}^{-1}+\cdots+p_{m}^{-1}$. Then $P_{m+1}^{(k)}$ is is bounded from $L^{p_{0}}\left(\mathbb{R}^{d}\right) \times$ $L^{p_{1}}\left(\mathbb{R}^{d}\right) \times \cdots \times L^{p_{m}}\left(\mathbb{R}^{d}\right)$ to $L^{p, \infty}\left(\mathbb{R}^{d}\right)$ when $1 \leq p_{j}<\infty$ and into $L^{p}\left(\mathbb{R}^{d}\right)$ when $1<p_{j}<\infty$ for all $j$.

We will need the following lemma, which is Corollary 4 in [11].

Lemma 1.2. Let $\Psi$ be a smooth bump whose Fourier transform is supported in an annulus that does not contain the origin and satisfies, for some positive integer $q$,

$$
\sum_{j \in \mathbb{Z}} \widehat{\Psi}\left(2^{-j q} \xi\right)=1, \quad \xi \in \mathbb{R}^{d} \backslash\{0\} .
$$

Then for any $0<p<\infty$ there is a constant $C_{p, d}$ (that also depends on $\Psi$ ) such that for all functions $g$ in $L^{2}$ we have

$$
\|g\|_{L^{p, \infty}} \leq C_{p, d}\left\|\mathbf{S}_{q}^{\Psi}(g)\right\|_{L^{p, \infty}} .
$$

\section{The proof of the Theorem 1.1}

When all $p_{j}>1$, the fact $P_{m+1}^{(k)}: L^{p_{0}} \times L^{p_{1}} \times \cdots \times L^{p_{m}} \rightarrow L^{p}$ is a consequence of the corresponding weak type estimate via multilinear interpolation, see [10]. It will therefore suffice to prove that $P_{m+1}^{(k)}$ maps $L^{p_{0}} \times L^{p_{1}} \times \cdots \times L^{p_{m}}$ to $L^{p, \infty}$ when $1 /(m+1) \leq p<\infty$. 
We suppose that the Fourier transform of $\Theta$ is supported in the annulus $a_{0}<$ $|\xi|<b_{0}$ for some $0<a_{0}<b_{0}<\infty$, of $\Theta_{j}$ is supported in the annulus $a_{j}<|\xi|<b_{j}$ for some $0<a_{j}<b_{j}<\infty, 1 \leq j \leq m$.

Case 1: $m \geq 1$ and $k=m-1$.

Subcase 1.a: $m \geq 2$.

When $k=m-1$ only one partial sum operator $S_{j}$ appears in the product in (1.1). Then, for $m \geq 2, P_{m}^{(m-1)}\left(f, f_{1}, \ldots, f_{m}\right)$ is pointwise bounded by

$$
\left(\sum_{j \in \mathbb{Z}}\left|\Delta_{j}^{\Theta_{1}}\left(f_{1}\right) \cdots \Delta_{j}^{\Theta_{m-1}}\left(f_{m-1}\right)\right|^{2}\right)^{1 / 2}\left(\sum_{j \in \mathbb{Z}}\left|\Delta_{j}^{\Theta}\left(f_{0}\right) S_{j}^{\Theta_{m}}\left(f_{m}\right)\right|^{2}\right)^{1 / 2} .
$$

This is in turn controlled by

$$
\mathbf{S}^{\Theta_{1}}\left(f_{1}\right)\left[\mathbf{M}\left(f_{2}\right) \cdots \mathbf{M}\left(f_{m-1}\right)\right]\left[\mathbf{S}^{\Theta}\left(f_{0}\right) \mathbf{M}\left(f_{m}\right)\right]
$$

(with the understanding that the middle factor does not appear when $m=2$ ) which is easily shown to satisfy the claimed conclusion, by applying Hölder's inequality on weak $L^{p}$ spaces (i.e., $\left\|g_{0} g_{1} \cdots g_{m}\right\|_{L^{p, \infty}} \leq\left\|g_{0}\right\|_{L^{p_{0}, \infty}}\left\|g_{1}\right\|_{L^{p_{1}, \infty}} \cdots\left\|g_{m}\right\|_{L^{p_{m}, \infty}}$ ) and using the boundedness of the maximal and square functions from $L^{r}$ to $L^{r, \infty}$ for $1 \leq r<\infty$.

Subcase 1.b: $m=1$.

In this case we write

$$
S_{j}^{\Theta_{1}}=S_{j+r_{0}}^{\Theta_{1}}+\sum_{i=j+r_{0}+1}^{j} \Delta_{i}^{\Theta_{1}}
$$

for some $r_{0}<0$ chosen so that the spectra of $S_{j+r_{0}}^{\Theta_{1}}$ and $\Delta_{j}^{\Theta}$ are disjoint; picking $r_{0}$ so that $b_{1} 2^{r_{0}+j}<a_{0} 2^{j}$ suffices. Then the function $\Delta_{j}^{\Theta}(f) S_{j+r_{0}}^{\Theta_{1}}\left(f_{1}\right)$ is supported in the annulus

$$
\left(a_{0}-b_{1} 2^{r_{0}}\right) 2^{j}<|\xi|<\left(b_{0}+b_{1} 2^{r_{0}}\right) 2^{j} .
$$

We pick integers $n_{0}<m_{0}$ such that

$$
2^{n_{0}}<a_{0}-b_{1} 2^{r_{0}}<b_{0}+b_{1} 2^{r_{0}}<2^{m_{0}}
$$

and we choose a function $\Omega$ whose Fourier transform equals 1 on the annulus $2^{n_{0}}<|\xi|<2^{m_{0}}$, vanishes off the annulus $2^{n_{0}-1}<|\xi|<2^{m_{0}+1}$, and satisfies

$$
\sum_{\ell \in \mathbb{Z}} \widehat{\Omega}\left(2^{\left(m_{0}-n_{0}+1\right) \ell} \xi\right)=1, \quad \xi \in \mathbb{R}^{d} \backslash\{0\}
$$

It follows from (2.2) that

$$
\sum_{\ell \in \mathbb{Z}} \widehat{\Omega}\left(2^{\ell} \xi\right)=m_{0}-n_{0}+1, \quad \xi \in \mathbb{R}^{d} \backslash\{0\} .
$$


Then we write

$$
P_{2}^{(0)}\left(f_{0}, f_{1}\right)=\sum_{j \in \mathbb{Z}} \Delta_{j}^{\Omega}\left(\Delta_{j}^{\Theta}\left(f_{0}\right) S_{j+r_{0}}^{\Theta_{1}}\left(f_{1}\right)\right)+E,
$$

where $E$ is a finite sum of terms of the form $\sum_{j} \Delta_{j}^{\Theta}\left(f_{0}\right) \Delta_{j+c}^{\Theta_{1}}\left(f_{1}\right)$. Since $E$ is pointwise bounded by a constant multiple of $\mathbf{S}^{\Theta}\left(f_{0}\right) \mathbf{S}^{\Theta_{1}}\left(f_{1}\right)$, the required conclusion follows for $E$ via an application of Hölder's inequality for weak type spaces.

We need to argue a bit more to handle the first term on the right in (2.4). We pick a function $\Psi$ whose Fourier transform is equal to 1 on the annulus $2^{n_{0}-2}<$ $|\xi|<2^{m_{0}+2}$ and vanishes off the annulus $2^{n_{0}-3}<|\xi|<2^{m_{0}+3}$. Set $q=m_{0}-n_{0}+5$. We split $\mathbb{Z}$ as a disjoint union of sets $I_{s}=\{\ell q+s, \ell \in \mathbb{Z}\}, 0 \leq s \leq q-1$. Next we split the sum in (2.4) as a finite sum over $s \in\{0,1, \ldots, q-1\}$ of the sums

$$
\Sigma_{s}=\sum_{j \in I_{s}} \Delta_{j}^{\Omega}\left[\Delta_{j}^{\Theta}\left(f_{0}\right) S_{j+r_{0}}^{\Theta_{1}}\left(f_{1}\right)\right]
$$

We also define a function $\Psi_{s}$ by setting $\widehat{\Psi_{s}}(\xi)=\widehat{\Psi}\left(2^{-s} \xi\right)$ and we note that $\sum_{\ell} \widehat{\Psi_{s}}\left(2^{-\ell q} \xi\right)=1$ for $\xi$ in $\mathbb{R}^{d} \backslash\{0\}$.

We make the following crucial observation: for $j \in I_{s}$ and $\ell \in \mathbb{Z}$ the supports of the functions $\xi \rightarrow \widehat{\Psi_{s}}\left(2^{-\ell q} \xi\right)$ and $\xi \rightarrow \widehat{\Omega}\left(2^{-j} \xi\right)$ intersect exactly when $j=\ell q+s$ and this case $\Delta_{j}^{\Omega} \Delta_{\ell q}^{\Psi_{s}}=\Delta_{j}^{\Omega}$ as the first function equals 1 on the support of the second. We deduce that for $j \in I_{s}$ and $\ell \in \mathbb{Z}$ we have

$$
\Delta_{\ell q}^{\Psi_{s}}\left[\sum_{j \in I_{s}} \Delta_{j}^{\Omega}\left[\Delta_{j}^{\Theta}\left(f_{0}\right) S_{j+r}^{\Theta_{1}}\left(f_{1}\right)\right]\right]=\Delta_{\ell q+s}^{\Omega}\left[\Delta_{\ell q+s}^{\Theta}\left(f_{0}\right) S_{\ell q+s+r_{0}}^{\Theta_{1}}\left(f_{1}\right)\right]
$$

and this exactly equals $\Delta_{\ell q+s}^{\Theta}(f) S_{\ell q+s+r_{0}}^{\Theta_{1}}\left(f_{1}\right)$. It follows that

$$
\mathbf{S}_{\mathbf{q}}^{\Psi_{\mathbf{s}}}\left(\Sigma_{s}\right)=\left(\sum_{\ell \in \mathbb{Z}}\left|\Delta_{\ell q}^{\Psi_{s}}\left(\Sigma_{s}\right)\right|^{2}\right)^{1 / 2}=\left(\sum_{\ell \in \mathbb{Z}}\left|\Delta_{\ell q+s}^{\Theta}\left(f_{0}\right) S_{\ell q+s+r_{0}}^{\Theta_{1}}\left(f_{1}\right)\right|^{2}\right)^{1 / 2}
$$

which is pointwise controlled by a constant multiple of $\mathbf{S}_{q}^{\Theta}\left(f_{0}\right) \mathbf{M}\left(f_{1}\right)$. To apply Lemma 1.2 we need to show that $\Sigma_{s}$ defined in (2.5) lies in $L^{2}$. By the orthogonality of $L^{2}$-norms, we have

$$
\begin{aligned}
\left\|\sum_{j \in I_{s}} \Delta_{j}^{\Omega}\left[, \Delta_{j}^{\Theta}\left(f_{0}\right) S_{j+r_{0}}^{\Theta_{1}}\left(f_{1}\right)\right]\right\|_{L^{2}}^{2} & =\sum_{j \in I_{s}} \int_{\mathbf{R}^{n}}\left|\Delta_{j}^{\Omega}\left[\Delta_{j}^{\Theta}\left(f_{0}\right) S_{j+r_{0}}^{\Theta_{1}}\left(f_{1}\right)\right](x)\right|^{2} d x \\
& \leq C\left\|M\left(f_{1}\right)\right\|_{L^{\infty}} \sum_{j \in I_{s}} \int_{\mathbf{R}^{n}}\left|\Delta_{j}^{\Theta}\left(f_{0}\right)(x)\right|^{2} d x \\
& \leq C\left\|f_{1}\right\|_{L^{\infty}}\left\|f_{0}\right\|_{L^{2}}^{2}<\infty .
\end{aligned}
$$

Using Lemma 1.2, for each $s \in\{0,1, \ldots, q-1\}$ we obtain that

$$
\left\|\Sigma_{s}\right\|_{L^{p, \infty}} \leq C_{p}\left\|\mathbf{S}_{\mathbf{q}}^{\Psi_{\mathbf{s}}}\left(\Sigma_{s}\right)\right\|_{L^{p, \infty}}
$$


and by the previous discussion this expression at most a constant multiple of $\left\|\mathbf{S}_{q}^{\Theta}\left(f_{0}\right) \mathbf{M}\left(f_{1}\right)\right\|_{L^{p, \infty}}$. The required conclusion is an easy consequence of Hölder's inequality and of the boundedness of the maximal and square functions from $L^{r}$ to $L^{r, \infty}$ for $1 \leq r<\infty$.

Case 2: $m \geq 2$ and $k<m-1$.

Having established the case $k=m-1$, we continue the proof by reverse induction on $k$. Fix a $k \in\{0,1, \ldots, m-2\}$ and assume that the conclusion is valid for all $k^{\prime}>k$ (and $k^{\prime} \leq m-1$.) We need to prove the same conclusion for $k$.

We begin by writing for all $s \in\{k+1, \ldots, m\}$

$$
S_{j}^{\Theta_{s}}=S_{j+r_{s}}^{\Theta_{s}}+\sum_{i=j+r_{s}+1}^{j} \Delta_{i}^{\Theta_{s}}
$$

for some $r_{s}<0$ that satisfy

$$
b_{k+1} 2^{r_{k+1}}+\cdots+b_{m} 2^{r_{m}}<a_{0}
$$

so that the spectra of $S_{j+r_{k+1}}^{\Theta_{k+1}}\left(f_{k+1}\right) \cdots S_{j+r_{m}}^{\Theta_{m}}\left(f_{m}\right)$ and $\Delta_{j}^{\Theta}\left(f_{0}\right)$ are disjoint.

Then we express $P_{m+1}^{(k)}$ as a finite sum of operators of the form $P_{m+1}^{(k+1)}, P_{m+1}^{(k+2)}$, $\ldots, P_{m+1}^{(m-1)}$ plus

$$
\sum_{j \in \mathbb{Z}}\left[\Delta_{j}^{\Theta}\left(f_{0}\right) \prod_{s=k+1}^{m} S_{j+r_{s}}^{\Theta_{s}}\left(f_{s}\right)\right]\left[\prod_{s=1}^{k} \Delta_{j}^{\Theta_{s}}\left(f_{s}\right)\right],
$$

with the understanding that if $k=0$, the last product does not appear. The induction hypothesis on $k$ yields the boundedness of $P_{m+1}^{(k+1)}, P_{m+1}^{(k+2)}, \ldots, P_{m+1}^{(m-1)}$, while the boundedness of (2.7) is discussed below considering two subcases.

Subcase 2.a: $k \geq 1$.

In this subcase things are straightforward. We apply the Cauchy-Schwarz inequality to control (2.7) by the product of the $\ell^{2}$ norms of the expressions inside the square brackets and therefore by the product

$$
\mathbf{S}^{\Theta}\left(f_{0}\right) \mathbf{S}^{\Theta_{1}}\left(f_{1}\right)\left[\prod_{s=2}^{m} \mathbf{M}\left(f_{s}\right)\right] .
$$

Obviously, this expression is bounded from $L^{p_{0}} \times \cdots \times L^{p_{m}}$ to $L^{p, \infty}$.

Subcase 2.b: $k=0$.

Condition (2.6) implies that the function $\Delta_{j}^{\Theta}\left(f_{0}\right) S_{j+r_{1}}^{\Theta_{1}}\left(f_{1}\right) \cdots S_{j+r_{m}}^{\Theta_{m}}\left(f_{m}\right)$ is supported in the annulus $2^{n_{0}} 2^{j}<|\xi|<2^{m_{0}} 2^{j}$ where $n_{0}<m_{0}$ are integers chosen so that

$$
2^{n_{0}}<\left(a_{0}-\left(b_{1} 2^{r_{1}}+\cdots+b_{m} 2^{r_{m}}\right)\right)<\left(b_{0}+b_{1} 2^{r_{1}}+\cdots+b_{m} 2^{r_{m}}\right)<2^{m_{0}} .
$$


We choose a smooth function $\Omega$ which is equal to 1 on the annulus $2^{n_{0}}<$ $|\xi|<2^{m_{0}}$ and vanishes off the annulus $2^{n_{0}-1}<|\xi|<2^{m_{0}+1}$. Then we write the expression in (2.7) as follows:

$$
\sum_{j \in \mathbb{Z}} \Delta_{j}^{\Omega}\left[\Delta^{\Theta}\left(f_{0}\right) \prod_{s=1}^{m} S_{j+r_{s}}^{\Theta_{s}}\left(f_{s}\right)\right] .
$$

We now pick a function $\Psi$ whose Fourier transform is equal to 1 on the annulus $2^{n_{0}-2}<|\xi|<2^{m_{0}+2}$ and vanishes outside the annulus $2^{n_{0}-3}<|\xi|<2^{m_{0}+3}$. Set $q=m_{0}-n_{0}+5$. We split $\mathbb{Z}$ as a disjoint union of sets $I_{s}=\{\ell q+s, \ell \in \mathbb{Z}\}$, $0 \leq s \leq q-1$. Next we split the sum in (2.8) as a finite sum over $s \in\{0,1, \ldots, q-1\}$ of the sums $\Sigma_{s}$ where the indices $j$ in (2.8) run over the set $I_{s}$. We also define a function $\Psi_{s}$ by setting $\widehat{\Psi_{s}}(\xi)=\widehat{\Psi}\left(2^{-s} \xi\right)$ and we note that $\sum_{\ell} \widehat{\Psi_{s}}\left(2^{-\ell q} \xi\right)=1$ for $\xi$ in $\mathbb{R}^{d} \backslash\{0\}$.

We observe that for $j \in I_{s}$ and $\ell \in \mathbb{Z}$ the supports of the functions $\xi \rightarrow$ $\widehat{\Psi_{s}}\left(2^{-\ell q} \xi\right)$ and $\xi \rightarrow \widehat{\Omega}\left(2^{-j} \xi\right)$ intersect nontrivially exactly when $j=\ell q+s$ and this case $\Delta_{j}^{\Omega} \Delta_{\ell q}^{\Psi_{s}}=\Delta_{j}^{\Omega}$. We are therefore in a position to use Lemma 1.2 , since again we can control the $L^{2}$-norm of $\sum_{j \in I_{s}} \Delta_{j}^{\Omega}\left[\Delta^{\Theta}\left(f_{0}\right) \prod_{s=1}^{m} S_{j+r_{s}}^{\Theta_{s}}\left(f_{s}\right)\right]$ by $C \prod_{s=1}^{m}\left\|f_{s}\right\|_{L^{\infty}}\left\|f_{0}\right\|_{L^{2}}<\infty$, and argue as in Subcase 2.2 to complete the proof.

Remark 2.1. The exponent $p_{j}$ can be taken to be equal to infinity whenever the maximal function $\mathbf{M}\left(f_{j}\right)$ appears in the estimate controlling $P_{m+1}^{(k)}$ (pointwise or in norm). For instance, when $m \geq 2$ and $k=m-1$, we may take $p_{2}=\cdots=p_{m}=\infty$; see (2.1).

\section{References}

[1] Auscher, P., Hofmann, S., Muscalu, C., Tao, T. and Thiele, C.: Carleson measures, trees, extrapolation and $T b$ theorems. Publ. Mat. 46 (2002), no. 2, $257-325$.

[2] Bényi, A., Maldonado, D., Nahmod, A. R. and Torres, R. H.: Bilinear paraproducts revisited. Math. Nachr. 283 (2010), no. 9, 1257-1276.

[3] BÉnyi, A., Maldonado, D. And Naibo, V.: What is a paraproduct? Notices Amer. Math. Soc. 57 (2010), no. 7, 858-860.

[4] Bernicot, F.: Uniform estimates for paraproducts and related multilinear multipliers. Rev. Mat. Iberoam. 25 (2009), no. 3, 1055-1088.

[5] Bony, J. M.: Calcul symbolique et propagation des singularités pour les équations aux dérivées partielles non linéaires. Ann. Sci. École Norm. Sup. (4) 14 (1981), no. 2 , 209-246.

[6] Coifman, R. And Meyer, Y.: Au délà des opérateurs pseudo-differentiels. Astérisque 57, Société Mathématique de France, Paris, 1978.

[7] David, G. And Journé, J. L.: A boundedness criterion for generalized CalderónZygmund operators. Ann. of Math. (2) 120 (1984), no. 2, 371-397.

[8] Grafakos, L.: Classical Fourier analysis. Graduate Texts in Mathematics 249, Springer, New York, 2008. 
[9] Grafakos, L. and Kalton, N. J.: The Marcinkiewicz multiplier condition for bilinear operators. Studia Math. 146 (2001), no. 2, 115-156.

[10] Grafakos, L., Liu, L., Lu, S. And Zhao, F.: The multilinear Marcinkiewicz interpolation theorem revisited: the behavior of the constant. J. Funct. Anal. 262 (2012), no. 5, 2289-2313.

[11] He, D.: Square function characterization of weak Hardy spaces. J. Fourier Anal. Appl. 20 (2014), no. 5, 1083-1110.

[12] Lacey, M. And Metcalfe, J.: Paraproducts in one and several parameters. Forum Math. 19 (2007), no. 2, 325-351.

[13] Muscalu, C., Pipher, J., Tao, T. and Thiele, C.: Bi-parameter paraproducts. Acta Math. 193 (2004), no. 2, 269-296.

[14] Muscalu, C., Pipher, J., Tao, T. and Thiele, C.: Multi-parameter paraproducts. Rev. Mat. Iberoam. 22 (2006), no. 3, 963-976.

[15] Sato, S. And Yabuta, K.: Multilinearized Littlewood-Paley operators. Sci. Math. Jpn. 55 (2002), no. 3, 447-453.

[16] Yabuta, K.: A multilinearization of Littlewood-Paley's $g$-function and Carleson measures. Tôhoku Math. J. 34 (1982), no. 2, 251-275.

Received May 16, 2013.

Loukas Grafakos: Department of Mathematics, University of Missouri, Columbia, MO 65211, USA.

E-mail: grafakosl@missouri.edu

DANQIng He: Department of Mathematics, University of Missouri, Columbia, MO 65211, USA.

E-mail: dhd27@mail.missouri.edu

Nigel Kalton: Department of Mathematics, University of Missouri, Columbia, MO 65211, USA.

Mieczyseaw MastyŁo: Faculty of Mathematics and Computer Science, A. Mickiewicz University; and Institute of Mathematics, Polish Academy of Science (Poznań branch), Umultowska 87, 61-614 Poznań, Poland.

E-mail: mastylo@math.amu.edu.pl

The first author acknowledges the support of the grant DMS 0900946. The third author deceased on August 31, 2010. The last named author was supported by the Foundation for Polish Science (FNP). 